highlight the exciting opportunities for better diagnosis and care of cancer patients.

\section{MOLECULAR GENETICS OF NON-SMALL CELL LUNG CANCER - CURRENT STATE OF PLAY}

Wendy A. Cooper ${ }^{1,2,3}$

${ }^{1}$ Tissue Pathology and Diagnostic Oncology, Royal Prince Alfred Hospital, Camperdown, ${ }^{2}$ Sydney Medical School, University of Sydney, and ${ }^{3}$ School of Medicine, University of Western Sydney, NSW, Australia

Pathologists are uniquely positioned to provide comprehensive morphological, immunophenotypic and molecular characterisation of lung cancers, which enables treatment to be optimally tailored for each individual patient. Driver mutations have been identified in over $50 \%$ of NSCLC but currently, only a subset of these can be targeted clinically. In the Australian population, activating mutations in epidermal growth factor receptor $(E G F R)$ gene are found in approximately $15 \%$ of lung adenocarcinomas while rearrangements of the $A L K$ gene occur in $<5 \%$ and assessment of these genes in all non-squamous NSCLC is essential for patient access to targeted therapies. Numerous additional targeted therapies are in clinical development including new generation EGFR tyrosine kinase inhibitors that require identification of acquired resistance mutations such as T790M. Assessment of programmed death-ligand 1 (PD-L1) provides information on the immune modulating status of a tumour which helps predict the likelihood of a patient responding to monoclonal antibodies against PD-1/PD-L1, a new class of therapy showing promise in all subtypes of NSCLC. Pathologists are instrumental in developing and evaluating appropriate molecular predictive markers to identify the most appropriate patients for each targeted therapy and we must take responsibility for ensuring accurate molecular characterisation is provided for all patients.

\section{A STANDARDISED APPROACH TO THYROID CYTOLOGY}

M. Priyanthi Kumarasinghe

PathWest and School of Pathology and Laboratory Medicine, Perth, WA, Australia

A standardised approach to thyroid cytology handling and reporting was recently endorsed by the Royal College of Pathologists of Australasia (RCPA). The aims were to provide a comprehensive description of appropriate collection and preparation of thyroid FNA material to optimise accurate assessment and reporting and to develop a structred system for standardised reporting of FNA cytology of the thyroid. The
Bethesda System for Reporting Thyroid Cytopathology (TBSRTC) has gained wide acceptance in the United States and elsewhere while there have been a few systems of terminology and reporting of thyroid cytology in use in Australasia over the last decade. The recommended terminology is based on the TBSRTC categories with some minor modifications and strong recommendations, taking into consideration the existing local healthcare delivery structure and past and current thyroid cytology practice.

Standard reporting includes a general classification (category descriptor) followed by a specific diagnosis (confirmed, favoured or suggested). A category descriptor (Non diagnostic; Benign; Indeterminate or follicular lesions of undetermined significance; Suggestive of a follicular neoplasm; Suspicious of malignancy; Malignant) should always be stated in the report in conjunction with or without the category number when using this classification system. The category number should not be used without the descriptor, to avoid any potential miscommunication or confusion with numbered categories used for other sites, e.g. breast. A standard recommendation for follow-up may be used, particularly when referring clinicians are not specialists in the field.

\section{STANDARDISED TERMINOLOGY IN PANCREATICOBILIARY CYTOLOGY}

$\underline{\text { Peter Earls }}$

Department of Anatomical Pathology, St Vincent's Hospital, Darlinghurst, NSW, Australia

The Papanicolaou Society of Cytopathology has recently published a reporting system for reporting pancreaticobiliary cytology. ${ }^{1}$ This is a six tiered system:

Category I: Non-diagnostic

Category II: Negative for malignancy

Category III: Atypical

Category IV: Neoplastic: benign; Neoplastic: other

Category V: Suspicious for malignancy

Category VI: Malignant

The system is a practical solution to achieving reporting consistency in this challenging area and its widespread adoption should lead to benefits in communication, quality, training, research and ultimately improved patient outcomes. Through the use of illustrative cases, this lecture will demonstrate its use in routine practice.

\section{References}

1. Pitman MB, Centeno BA, Ali SZ, et al. Standardized terminology and nomenclature for pancreatobiliary cytology: The Papanicolaou Society of Cytopathology Guidelines. Cytojournal 2014; 11 (Suppl 1): 3. http://www.cytojournal.com/ text.asp?2014/11/2/3/133343 